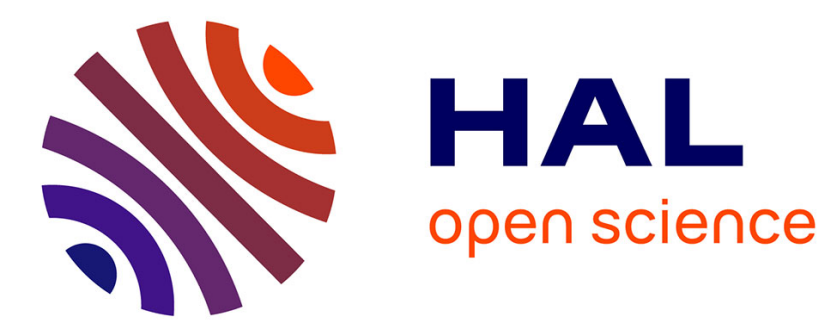

\title{
Système familial et attachement : revue de la question
}

S. Pinel-Jacquemin, Chantal Zaouche Gaudron

\section{To cite this version:}

S. Pinel-Jacquemin, Chantal Zaouche Gaudron. Système familial et attachement : revue de la question . Neuropsychiatrie de l'Enfance et de l'Adolescence, 2009, 57 (3), pp.167 - 172. 10.1016/j.neurenf.2008.09.006 . hal-01498538

\section{HAL Id: hal-01498538 \\ https://hal-univ-tlse2.archives-ouvertes.fr/hal-01498538}

Submitted on 28 Apr 2017

HAL is a multi-disciplinary open access archive for the deposit and dissemination of scientific research documents, whether they are published or not. The documents may come from teaching and research institutions in France or abroad, or from public or private research centers.
L'archive ouverte pluridisciplinaire HAL, est destinée au dépôt et à la diffusion de documents scientifiques de niveau recherche, publiés ou non, émanant des établissements d'enseignement et de recherche français ou étrangers, des laboratoires publics ou privés. 


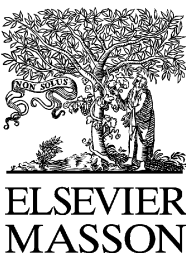

Disponible en ligne sur

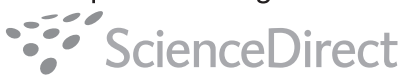

www.sciencedirect.com
Elsevier Masson France

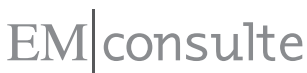

www.em-consulte.com

Neuropsychiatrie de l'enfance et de l'adolescence xxx (2008) xxx-xxx

neuropsychiatrie

de l'enfance

et de l'adolescence

Article original

\title{
Système familial et attachement : revue de la question
} Family system and attachment: Question review

\author{
S. Pinel-Jacquemin ${ }^{\mathrm{a}, *}$, C. Zaouche-Gaudron ${ }^{\mathrm{b}}$ \\ a Laboratoire psychologie du développement et processus de socialisation, équipe «Milieux, groupes et psychologie du jeune enfant», \\ université Toulouse, UTM, 5, allées Antonio-Machado, 31058 Toulouse cedex 9, France \\ ${ }^{\mathrm{b}}$ Université de Toulouse, UTM, France
}

\section{Résumé}

L'attachement parent-enfant constitue un lien affectif entre l'enfant et sa mère ou son père, qui lui permet d'utiliser celle-ci, ou celui-ci, comme un port de sécurité lorsqu'il est en situation de détresse, puis comme une base de sécurité à partir de laquelle il peut à nouveau explorer lorsque le danger a disparu. D'abord focalisée sur la dyade mère-enfant, la théorie de l'attachement s'est ensuite étendue à celle constituée par le père et son enfant, puis à un ensemble de figures proches de l'enfant (frères, sœurs, grands-parents, pairs, etc.). Aujourd'hui, plus de 30 ans après sa validation empirique initiale, l'attachement parent-enfant interroge encore la communauté scientifique. Parallèlement, la théorie du système familial nous enseigne que, constituée d'éléments en interaction, la famille, premier milieu dans lequel baigne l'enfant, fonctionne comme un système. Ainsi la jonction entre ces deux modèles théoriques est-elle largement souhaitée et nombreux sont les auteurs qui la sollicitent afin de comprendre l'influence des relations intrafamiliales sur la qualité de l'attachement parent-enfant. Ces relations incluent les relations conjugales, coparentales, parent-enfant et les relations fraternelles. Nous nous proposons ici de faire le point sur l'ensemble des recherches qui ont étudié l'impact de chacune d'elles sur la qualité de l'attachement parent-enfant. Ce travail s'inscrit dans une perspective écosystémique et constitue une étape importante vers une approche systémique de l'attachement, prenant en compte la dynamique de l'ensemble des relations familiales.

() 2008 Elsevier Masson SAS. Tous droits réservés.

\section{Abstract}

The parent-child attachment is an emotional bond between the child and his mother, or his father, who allows it to use her, or him, as a safety haven, when in distress, and then as a security base from which it can explore again when the danger has disappeared. Initially focused on the mother-child dyad, attachment theory then spread to the one established by the father and his child, then a set of figures closed to the child (brothers, sisters, grandparents, peers, and so on.). Today, more than 30 years after its initial empirical validation, the parent-child attachment is still interviewing scientists. Meanwhile, another theory involves research in psychology, the family system. Consisting of interacting elements, the family functions as a system and is also the first milieu in which steeps the child. The junction between the two theories is widely desired, and many are the authors who seek to understand the influence of intra-family relations on the quality of parent-child attachment. These relationships include marital relations, co-parenting, parent-child and sibling relationships. We propose here to take stock of all the researches that have studied the impact of each of them on the quality of parent-child attachment. This work is part of an eco-systemic perspective and is an important step toward a systemic approach, taking into account the dynamics of all family relationships.

(ㅇ) 2008 Elsevier Masson SAS. Tous droits réservés.

Mots clés : Attachement parent-enfant ; Approche systémique ; Relations conjugales ; Relations coparentales ; Relations parent-enfant ; Relations fraternelles Keywords: Parent-child attachment; Systemic approach; Marital relationships; Co-parental relationships; Parent-child relationships; Sibling relationships

\section{* Auteur correspondant}

Adresse e-mail : steph.jacquemin@worldonline.fr (S. Pinel-Jacquemin).

\section{Introduction}

La théorie de l'attachement [1], au niveau du développement de l'enfant, a été explorée, depuis plus de 30 ans, tant sur les plans affectif et émotionnel, que sur les plans social et cognitif. Les relations d'attachement parent-enfant constituent un lien 
42 affectif entre l'enfant et son parent. En situation de détresse, l'enfant utilise sa figure d'attachement comme base de sécurité pour assurer sa protection et pouvoir ainsi à nouveau explorer son environnement. Si Bowlby [1] admet la multiplicité des figures d'attachement, la mère reste la figure principale. Selon le degré de manifestations d'anxiété ou d'évitement exprimé lors des retrouvailles mère-enfant, l'attachement est classé sécurisé, anxieux-évitant, anxieux-résistant ou ambivalent ou encore désorganisé-désorienté [2]. Ce besoin de sécurité est guidé par un modèle interne opérant (MIO), représentation mentale, consciente et inconsciente, du monde extérieur et de soi à l'intérieur de ce monde, constituant un schéma mental pour les relations sociales futures de l'enfant.

Parmi les questions qui restent posées encore aujourd'hui à partir de ces données princeps, celle du rôle joué par le contexte dans lequel prend naissance cet attachement, et en particulier le contexte familial, apparaît comme centrale. La nécessité de « recontextualiser» l'attachement dyadique «figure d'attachement-enfant» dans le milieu familial dans lequel il se constitue, fait maintenant l'unanimité des chercheurs $[3,4]$. Bowlby [5], lui-même, revint, au terme de son élaboration théorique, sur la nécessité d'intégrer la théorie de l'attachement à l'ensemble du système familial. L'enfant doit, en effet, s'adapter à une famille dont les éléments, réunis en sous-systèmes, l'influencent à la fois directement, à travers leurs interactions avec lui, et, indirectement, à travers leurs interactions entre eux [6]. Byng-Hall [7] a ainsi proposé une extension de la notion de base de sécurité à la famille comme un tout. Dans une perspective clinique, il a souligné l'importance pour l'enfant d'un réseau familial fiable et d'une «base de sécurité familiale» avec une conscience partagée par les membres de la famille que l'attachement doit être protégé. Marvin et Stewart [8] proposent, quant à eux, la notion de « MIO partagés » par la famille comme des croyances communes et des règles sur les comportements analogues aux membres de la famille. Tout cadre commun peut être vu comme un «état d'esprit partagé » qui permet aux membres de la famille de répondre de façon adéquate aux besoins d'attachement, de discipline et de sécurité de chacun d'entre eux [9]. Cependant chaque enfant se forme des représentations des systèmes familiaux qui sont basées sur une perspective composite du climat socio-émotionnel familial et dues à ses expériences avec les multiples sous-systèmes familiaux [10]. La perception de la sécurité qu'élabore l'enfant est le produit des expériences collectives qu'il vit à l'intérieur de ses multiples relations familiales [11]. Le système familial représente donc une réalité différente pour chacun de ses membres [12], ce qui peut alors expliquer que deux enfants d'une même fratrie aient un attachement parent-enfant différent.

$\mathrm{Au}$ sein de la famille nucléaire, les relations intrafamiliales comportent plusieurs relations : conjugales, coparentales, parent-enfant et enfin fraternelles. Plusieurs études font état de relations de causalité de nature linéaire et amènent ainsi une vision déterministe de l'attachement. La théorie générale des systèmes (van Bertalanffy, 1968) nous enseigne que «le tout est différent de la somme des parties ». La majorité des études citées ci-après sont issues de la base de données PsycINFO qui regroupe, à ce jour, près de 2,3 millions de références en lien avec la psychologie et les uiscrpmines commexes, cont No,563 sur l'attachement, en 25 langues. Notre revue de la question s'est effectuée par mots clés ${ }^{1}$ reprenant de manière systématique le nom du sous-système considéré et l'attachement (exemple : « relations conjugales » et «attachement»). Nous avons privilégié les travaux prenant en compte des enfants de six à 12 ans, période développementale où le $\mathrm{MIO}$ de l'enfant est stabilisé et où l'enfant a encore de nombreuses relations à l'intérieur de sa famille. Au total, à partir de plus de 500 références passées en revue pour la plupart anglo-saxonnes mais aussi françaises (ou francophones) et européennes, nous avons retenu celles qui illustrent de façon précise la problématique envisagée dans cette contribution (environ une cinquantaine selon les normes de publication demandées).

\section{Système familial et attachement}

\subsection{Les relations conjugales et la qualité de l'attachement parent-enfant}

Le sous-système conjugal est un sous-système intragénérationnel composé des deux conjoints à l'origine de la création de la famille. Il ressort de la littérature que trois composantes importantes de ce sous-système ont une influence sur la qualité de l'attachement parent-enfant : la représentation d'attachement, la satisfaction conjugale de chacun des conjoints et les conflits conjugaux, en particulier tels qu'ils sont perçus par les enfants.

\subsubsection{La représentation d'attachement des conjoints}

Chacun des conjoints, étant lui-même issu d'un système familial qui lui est propre, s'est forgé une représentation d'attachement, un MIO, de ses relations avec ses propres parents dans son enfance. La forte incidence de cette représentation d'attachement sur la qualité de l'attachement de l'enfant a été mise en évidence, entre la classification réalisée à partir de l'Adult Attachment Interview de la mère (autonome, détaché, préoccupé ou désorganisé) et l'estimation de la classification d'attachement mère-enfant lors de la Situation Étrange (sécurisé, ambivalent, anxieux-évitant ou désorganisé). [2]. Ce mécanisme de transmission s'expliquerait - du moins partiellement - par l'influence des modèles d'attachement du parent sur sa sensibilité envers l'enfant. Cette sensibilité influencerait la qualité des interactions et favoriserait le sentiment de sécurité de l'enfant. Plusieurs études ont montré que les MIO des parents étaient liés à leurs comportements dans l'interaction avec leurs enfants [13]. Steele et al. [14] parlent d' «effet primaire d'attachement maternel» en montrant que cette transmission intergénérationnelle serait le fait de la mère [9] et non du père ou des deux parents. Cet effet serait à nuancer selon le sexe des enfants puisque la classification d'attachement des filles serait

\footnotetext{
1 Relations conjugales, relations coparentales, relations père-enfant, relations mère-enfant, relations fraternelles, attachement, puis satisfaction conjugale, conflits conjugaux, alliance coparentale, traitement différentiel parental, style éducatif, tempérament, sexes des enfants et attachement, etc.
} 
liée de manière significative à la classification de leurs deux parents alors que ce ne serait pas le cas des garçons [15].

\subsubsection{La satisfaction conjugale}

La satisfaction conjugale est définie comme la qualité des relations conjugales. Elle englobe la communication et les échanges/partages dans le couple ainsi que la représentation de bonheur et de réussite conjugale des deux partenaires. Les études montrent qu'elle prédit la qualité de l'attachement père-enfant, mais pas celle de l'attachement mère-enfant [16]. La synchronie, c'est-à-dire la façon dont une interaction apparait être réciproque et mutuelle [17], est un médiateur entre la satisfaction conjugale et l'attachement père-enfant et entre les symptômes dépressifs de la mère et l'attachement mère-enfant [16].

\subsubsection{Les conflits conjugaux}

Les dimensions de disponibilité émotionnelle parentale, telles que la chaleur, la sensibilité, le soutien, l'hostilité, servent en partie de médiateurs entre le conflit conjugal et la sécurité d'attachement parent-enfant [18]. Il a été démontré que le conflit conjugal, perçu comme menaçant par l'enfant, prédit des relations d'attachement parent-enfant insécurisées et désorganisées, même après que soient contrôlées la chaleur et la sensibilité parentales [19]. L'étude de Davies et Forman, sur 56 enfants de six à neuf ans ( $\mu=7,5$ ans) ayant un attachement «évitant» ou « ambivalent», montre qu'ils vivent plus de conflit conjugal que les enfants « sécurisés » [19]. Cependant, un conflit conjugal croissant est directement lié à une insécurité d'attachement père-enfant mais pas à celle de l'attachement mère-enfant [20]. Certains auteurs expliquent cela par le fait que la paternité et la conjugalité seraient pour les pères une seule et même entité [21] et que la qualité des différentes relations familiales aurait plus d'impact pour eux que pour les mères. Pour autant, le conflit conjugal peut aussi être exprimé de façon constructive, par exemple, lors de sa résolution. Il peut être modéré, avoir lieu dans un environnement familial par ailleurs chaleureux et soutenant, et, dans ces cas, les enfants peuvent tirer des enseignements profitables sur la manière de négocier le conflit et le résoudre [22]. Soutien et sécurité apportés par de bonnes relations parent-enfant peuvent, le cas échéant, servir de protection aux enfants contre les effets des conflits interparentaux [23].

Ainsi, «il y a une continuité dans la qualité affective entre l'interaction maritale et l'interaction familiale, non seulement au niveau des dyades parent-enfant, mais aussi au niveau de la dyade parentale » [24].

\subsection{Les relations coparentales et la qualité de l'attachement parent-enfant}

La relation coparentale est un sous-système familial caractérisé par une dynamique de groupe incluant le père, la mère et l'enfant. Le terme d'alliance coparentale [25] fait référence au degré de coopération des parents dans leurs rôles parentaux, allant d'un soutien sans faille à l'ébranlement systématique des initiatives du partenaire et à sa déstabilisation.

Un coparentage fait de compétition entre les parents est associé à la perception du père et de la mère d'avoir une relation d'attachement parent-enfant moins sécurisée (attachement mesuré à l'aide du Q-Sort [26]), alors que le soutien du père par la mère est associé à une relation d'attachement mère-enfant plus sécurisée [27].

\subsection{Les relations parent-enfant et la qualité de l'attachement parent-enfant}

Plusieurs composantes entrent ici en jeu dans la mesure où l'histoire de chacun des parents ainsi que ses caractéristiques individuelles façonnent l'interaction avec l'enfant. Chaque parent emploie un style éducatif propre, issu de sa propre éducation, mais aussi construit tout au long de son histoire de vie. Les caractéristiques de l'enfant, comme son genre et son tempérament, vont intervenir dans l'interaction et, selon la manière dont le parent les perçoit et les reçoit, l'interaction s'en trouvera influencée d'une manière ou d'une autre, ce qui explique le traitement différentiel parental selon les enfants. Nous allons donc maintenant présenter en quoi ces composantes, le style éducatif parental, le traitement différentiel parental et chacune des caractéristiques à la fois des parents et des enfants, influencent la qualité de l'attachement parent-enfant.

\subsubsection{Le style éducatif parental}

Le style éducatif parental, généralement défini selon les deux axes [28] que sont le contrôle et le soutien, comprend quatre catégories : « autoritaire » (contrôle élevé, soutien faible), authoritative ou «démocratique» (contrôle élevé, soutien élevé), «permissif » (contrôle faible, soutien élevé) et «désengagé » (contrôle faible, soutien faible). Nair et Murray [29] indiquent, dans leur recherche sur 58 dyades mère-enfant de trois à six ans, que le style parental apporte une contribution directe à l'attachement (mesuré avec le Q-Sort [26]) et qu'il serait même un médiateur entre la santé psychique de la mère (stress, dépression, conflit) et la sécurité de l'attachement. Karavasilis al. [30] trouvent, dans leur étude sur 202 enfants de neuf à 11 ans et 212 enfants de dix à 14 ans, une association positive entre le style parental authoritative et l'attachement sécurisé tandis que le style permissif prédit l'attachement évitant. L'implication parentale, la garantie de l'autonomie psychique, et le contrôle comportemental de l'enfant sont liés à la sécurité d'attachement de ce dernier à la mi-enfance et à la préadolescence [31]. Le type de relation le plus néfaste sur le système d'attachement de l'enfant (mesuré avec l'éche'remplacer (e) par pontrôle inexistant ou le manque d remplacer (e) par perçute) par 1019 enfants de huit (s): perçu(s) auteurs, l'influence d'une relation soutenante par les parents sur le développement d'un attachement sécurisé vient du père pour les filles et de la mère pour les garçons. Cette relation soutenante englobe le respect de l'individuation de l'enfant, une implication positive de la part des parents et une attention centrée sur l'enfant.

De manière réciproque, la qualité de l'attachement influence les pratiques parentales et la perception que s'en font les enfants. En effet, les liens d'attachement se construisent très tôt, dès les premiers jours de vie et vont structurer les rapports ultérieurs entre parents et enfants. «Les déficits précoces de l'attachement 
vont entacher les relations parents/enfants et entraîner des difficultés d'obéissance et de conformité aux consignes parentales. L'hostilité qui sous-tend les rapports suscite des conflits et perturbe l'exercice du contrôle parental, ce qui se traduit par le retrait de l'affection, l'absence de supervision ou par l'usage de formes de contrôles contraignantes, punitives ou coercitives » [34].

\subsubsection{Le traitement différentiel parental}

Sur 200 jeunes adultes, les sujets qui ont le sentiment d'avoir reçu une même qualité d'affection de leurs parents que leurs frères et sœurs ont des styles d'attachement plus sécurisés comparés aux individus ressentant une jalousie [35]. De même, les enfants qui se sentent «désavantagés » par rapport à leurs frères et sœurs ressentent moins d'attachement et de proximité à leurs parents que ceux qui se disent traités de manière équitable ou «favorisés» [36]. Il est important de noter que cette perception de légitimité ou non d'un certain «favoritisme» de la part des parents est davantage liée aux indices de bien-être socioémotionnel que l'amplitude du traitement différentiel parental en lui-même.

\subsubsection{Les caractéristiques individuelles des parents}

Nous proposons ci-après une description non exhaustive, mais qui se veut la plus complète possible, des résultats issus de la littérature scientifique. Parmi ces caractéristiques, nous évoquerons donc l'âge des parents, leur niveau d'éducation, le niveau socioéconomique, l'emploi de la mère puis les traits de personnalité des parents, la psychopathologie et enfin leurs pratiques éducatives.

2.3.3.1. L'âge des parents. Selon Payne [37], l'âge des parents n'est pas corrélé à la sécurité de l'attachement de leurs enfants, mesuré avec l'Attachment Behavior Q-Set, entre 12 et 18 mois.

\subsubsection{Le niveau d'éducation. Cet auteur ne trouve pas non} plus de lien significatif entre le niveau d'éducation des 47 parents interrogés et la sécurité d'attachement de leurs enfants. Caldera et Lindsey tempèrent ces résultats en montrant que ce serait dans les familles où le niveau d'études des pères est plus élevé que l'on trouve davantage d'attachements père-enfant sécurisés et davantage de mères capables de répondre à leur enfant de manière adéquate [27]. Dans les familles où les deux parents travaillent, il y a davantage de concordance d'attachement entre l'enfant et ses deux parents [27].

2.3.3.3. La reprise de l'emploi de la mère. La reprise de l'emploi de la mère n'empêche pas la formation de l'attachement. Les effets de l'emploi de la mère sont différents selon le sexe de l'enfant. On trouve davantage de garçons insécurisés (par rapport aux deux parents) dans les familles où la mère travaille que de filles insécurisées dans ces mêmes familles, ou que de filles et de garçons insécurisés dans les familles où la mère ne travaille pas [41]. D'autres auteurs trouvent, quant à eux, que le nombre d'heures de travail des parents n'est corrélé ni avec la sensibilité parentale, ni avec la qualité de l'attachement parent-enfant, mesuré pour le père et la mère avec la procédure de Situation Étrange lorsque l'enfant a respectivement 12 et 13 mois [42]. De même, dans cette étude, il n'y a pas d'influence significative du statut d'emploi maternel et du genre de l'enfant sur la sensibilité parentale. L'interaction entre le nombre d'heures de travail de la mère et la sensibilité parentale ne prédit pas la qualité de l'attachement de l'enfant à ses parents. Par ailleurs, une étude plus récente [43] vient confirmer que les mères plus engagées à combiner responsabilités professionnelles et familiales et moins anxieuses à l'idée de confier leur enfant (de 12 mois, dans l'étude) à quelqu'un de non familier sont plus susceptibles d'avoir des enfants sécurisés (attachement mesuré ici avec la procédure de la Situation Étrange) que les mères travaillant plus anxieuses.

2.3.3.4. Difficultés économiques. Dans les familles où les parents sont entravés par des difficultés économiques, ils sont moins sensibles et moins susceptibles de répondre aux besoins de leurs enfants [24].

\section{$\sqrt{ }$ Insérer: Les}

2.3.3.5. Traits de personnalité. Sur le registre des traits de personnalité, les expériences émotionnelles de la mère, les comportements expressifs (intérêt, joie, tristesse, dégoût, crainte) et la sociabilité ou l'empathie ont également été mis en évidence comme prédicteurs significatifs du niveau de sécurité de l'attachement mère-enfant, mesuré avec la Situation Étrange, (114 dyades mère-enfant de un an) [41]. Bigras et Paquette précisent que la sociabilité des mères est associée positivement à celle des filles plutôt qu'à celle des garçons [38], le processus d'identification favorisant l'acquisition chez l'enfant de certains comportements du parent de même sexe 40$]$.

\subsubsection{La symptomatologie parentale. La s 39}

remplacer 40 par

parentale, quant à elle, joue également un rôle dans la qualité de la relation d'attachement. Les enfants âgés de 36 mois développent davantage d'attachement insécurisé lorsque leur mère est déprimée [44]. Cela passerait par la relation parent-enfant, et notamment par la perception négative qu'a le parent déprimé de son enfant. On retrouve davantage de personnalités dépressives parmi les parents ayant une représentation d'attachement insécurisée et, par ailleurs, une représentation d'attachement sécurisée ne protège pas l'enfant de la perception négative qu'en a son parent dépressif [44].

2.3.3.7. Vratiques éducatives. Enfin, la cohérence ou l'incohérence d'un même parent dans ses pratiques éducatives et attitudes vis-à-vis de l'enfant est une dimension de la relation parent-enfant qui peut aussi jouer un rôle, en particulier, dans l'adaptation ou l'inadaptation émotionnelle de l'enfant [45] (45,ads supprimer la . Notons enfin, l'étude de Scher [ 4 virgule et mettre un fant dont l'attachement est mesuré l, espace

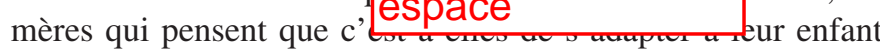
(«attitude facilitatrice») sont plus susceptibles d'avoir des enfants sécurisés que celles qui pensent que c'est à l'enfant de s'adapter à elles («attitude régulatrice »). 


\subsubsection{Les caractéristiques individuelles des enfants}

Outre la période développementale, l'influence de deux caractéristiques individuelles de l'enfant sur la qualité de l'attachement parent-enfant sont particulièrement traitées dans la littérature scientifique: le tempérament et le sexe.

\subsubsection{Le tempérament. Le tempérament oriente l'expression} émotionnelle de l'attachement, mais celui-ci se construit en fonction des réponses plus ou moins adaptées que l'enfant reçoit. Szewczyk-Sokolowski et al. (2005) [47] trouvent, sur 98 enfants, âgés de 54 mois en moyenne, dont l'attachement est mesuré par les réponses des mères au Q-Sort, une association significative et négative entre la sécurité de l'attachement et le tempérament difficile de l'enfant défini comme irritable, plus facilement contrarié et peu amène à se conformer aux demandes de ses parents. Les différences individuelles dans le tempérament et l'émotivité contribuent de manière significative au développement d'un attachement sécurisé ou non [40].

2.3.4.2. Le sexe de l'enfant. Au-delà du tempérament, le sexe de l'enfant est clairement associé à la sécurité de l'attachement paternel. On retrouve davantage d'attachements père-fils sécurisés que d'attachements père-fille, association non retrouvée pour l'attachement maternel [48].

Ainsi, il semblerait que les caractéristiques de l'enfant, sexe et tempérament, soient davantage associées au comportement paternel qu' au comportement maternel [49] et qu'elles exercent une influence particulière sur les relations entre le père et ses enfants.

Après avoir vu l'influence des relations conjugales, coparentales et parent-enfant sur la qualité de l'attachement parent-enfant, il nous reste maintenant à aborder celles de la fratrie.

\subsection{Les relations fraternelles et la qualité de l'attachement parent-enfant}

Ce sous-système intra-générationnel a non seulement sa dynamique propre, mais également une dynamique en relation avec les autres sous-systèmes, conjugal, parental, et, parents-enfants. En plus de la qualité des relations fraternelles, les composantes que nous avons mentionnées précédemment, à savoir le conflit conjugal, le style éducatif parental et le traitement différentiel parental exercent une influence sur les relations fraternelles, et donc potentiellement sur la sécurité émotionnelle des enfants. Certaines études ont analysé le lien entre l'attachement mère-enfant et la qualité des relations fraternelles. Elles ont, entre autres, montré les relations non conflictuelles qui unissent un aîné sécurisé, d'âge préscolaire, à son cadet sécurisé [52]. Une seule recherche, à notre connaissance, celle de Volling et al. [54], a étudié, sur 30 fratries (aîné : $\mu=72$ mois, cadet: $\mu=39,8$ mois), l'attachement père-enfant, mesuré avec la procédure de la Situation Étrange [55], et sa relation avec la qualité des relations fraternelles. Aucun lien entre ces deux variables n'a pu être trouvé.

Par ailleurs, selon que les frères et sœurs sont des étais les uns pour les autres, ils peuvent contribuer à augmenter la sécurité de leur environnement [50]. Stewart [51] et Teti et Ablard [52], en utilisant la procédure de la Situation Étrange, ont trouvé, qu'en l'absence des parents, le cadet pouvait se servir de l'aîné comme d'une base de sécurité à partir de laquelle explorer, et cela uniquement lorsque les deux enfants ont développé un attachement sécurisé à la mère. Ces résultats sont retrouvés dans une étude française plus récente [53], dans laquelle une plus grande sécurité du cadet par rapport à l'enfant unique est mise en évidence. L'hypothèse émise est qu'il existe une base de sécurité «fraternelle» spécifique et complémentaire de celle élaborée avec les deux parents.

\section{Conclusion}

Les résultats de ces études montrent que de multiples niveaux du fonctionnement familial, qu'ils soient dyadiques ou triadiques, influencent la qualité des relations d'attachement parents-enfants [18]. Chaque sous-système a non seulement un impact direct sur la qualité de l' attachement mais également indirect à travers ses liens avec l'ensemble des autres sous-systèmes. Cependant, et jusqu'à présent, chacun de ces résultats est interprété indépendamment des autres-ce qui peut d'ailleurs donner une vision déterministe du lien d'attachement-alors que nous savons que toutes les relations familiales, et plus particulièrement leur perception par chacun des membres impliqués dans la relation d'attachement, coexistent et s'auto-influencent.

Nous disposons, après cette revue de la littérature scientifique non exhaustive, d'un ensemble de facteurs contextuels, tous situés aux niveaux inter-, intra- et transgénérationnel, de la famille et ayant une influence sur la qualité de l'attachement parents-enfants. L'étude des processus compensatoires entre les sous-systèmes (par exemple, une relation conflictuelle compensée par une relation soutenante dans un autre soussystème), et/ou des processus spill-over (par exemple, une relation conflictuelle propagée dans un autre sous-système) permettra une meilleure compréhension, mais aussi une interprétation plus fine, de l'influence de la dynamique familiale globale sur la qualité des relations d'attachements père-enfant et mère-enfant. Une perspective s'ouvre alors, celle de pouvoir repérer les systèmes familiaux affectifs, caractérisés par les types d'attachement des enfants à leurs parents, selon les structures familiales, c'est-à-dire la qualité de chacune des interrelations à l'œuvre dans un système familial donné.

\section{Référence non citée}

\section{Références}

[1] Bowlby J., Attachement et perte. L'Attachement, vol. 1, Traduction française: Jeannine Kalmanovitch. Paris: PUF; 1978 (2 édition), 1969 ( $1^{\text {re }}$ édition)

[2] Main M, Kaplan N, Cassidy J.Security in infancy, childhood and adulthood: A move to the level of representation. In: In: Bretherton I, Waters E, editors. Growing points in attachment theory and research. Monographs of the Soc Res Child Dev, 50. 1985. p. 66-104. 
[3] Kozlowska K, Hanney L. The network perspective: an integration of attach-

[27] Caldera YM, Lindsey EW. Coparenting, mother-infant interaction, and ment and family systems theories. Fam Process 2002;41. insérer pages:

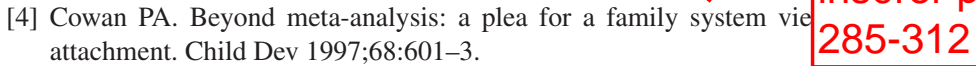

[5] Bowlby J. A secure base. Clinical applications of attachment theory. London: Routlegde; 1988.

[6] Lewis M. The Child and Its Family: The Social Network Model. Hum Dev, 2005, 48 (1-2), Special issue: Beyond the Dyad: Conceptualization of Social Networks. 8-27.

[7] Byng-Hall J. Family and couple therapy. In: Cassidy J, Shaver P, editors. Handbook of Attachment: Theory, Research and Clinical Implications. New York: Guilford Press; 1999. p. 625-45.

[8] Marvin RS, Stewart RB. A family system framework for the study of attachment. In: Greenberg MT, Chiccetti D, Cummings EM, editors. Attachment in the preschool years: Theory, Research and Intervention. Chicago: University of Chicago Press; 1990. p. 51-86.

[9] Hill J, Fonagy P, Safier E, Sargent J. The ecology of attachment in the family. Fam Process 2003;42:205-21.

[10] Cummings EM, Davies PT. Effect of marital conflict on children. J Psychol Psychiatry 2002;43:31-63.

[11] Ackerman BP, Izard CE, Schoff K, Youngstrom EA, Kogos J. Contextual risk, caregiver emotionality and the problem behaviors of six-and sevenyears-old children from economically disadvantaged families. Child Dev 1999;70:1415-27.

[12] Anderson SA, Sabatelli RM. Family interaction: A multigenerational developmental perspective. Boston, MA: Allyn \& Bacon; 1995.

[13] Cohn DA, Silver DH, Cowan CP, Cowan PA, Pearson J. Working models of childhood attachment and couple relationships. J Family Issues 1992;13:432-49.

[14] Steele H, Steele M, Fonagy P. Associations among attachment classifications of mothers, fathers and their parents. Child Dev 1996;67:54155.

[15] Owen MT, Easterbrooks MA, Chase-Lansdale PL. The relation between maternal employment status and the stability of attachments to mother and to father. Child Dev 1984;55:1894-901.

[16] Lundy BL. Paternal socio-psychological factors and infant attachment: the mediating role of synchrony in father-infant interactions. Infant Behav Dev 2002;25:221-36.

[17] Isabella RA, Belsky J, von Eye A. Origins of infant-mother attachment: an examination of interactional synchrony during the infant's first year. Dev Psychol 1978;25:12-21.

[18] Frosch CA, Mangelsdorf SC, McHale JL. Marital behaviour and the security of the preschooler-parent attachment relationships. J Fam Psychol 2000;14:144-61.

[19] Davies PT, Forman EM. Children's patterns of preserving emotional security in the interparental subsystem. Child Dev 2002;73(6):1880993.

[20] Owen MT, Cox MJ. Marital conflict and the development of infant-parent attachment relationships. J Fam Psychol 1997;11:152-64.

[21] Cohn DA, Cowan PA, Cowan CP, Pearson J. Mother's and father's working model of childhood attachment relationships, parenting styles and child behaviour. Dev Psychopathol 1992;4:417-31.

[22] Marc E, Picard D. L'École de Palo Alto, un nouveau regard sur les relations humaines ( $3^{\mathrm{e}}$ édition augmentée et enrichie). Paris: Retz; 2000. Coll. La Psychologie Dynamique.

[23] Emery RE. Interparental conflict and the children of discord and divorce. Psychol Bull 1982;92:310-30.

[24] Rouyer V, Devault A, Zaouche-Gaudron C. Les conditions de vie défavorisées influent-elles sur le développement des jeunes enfants? In: Zaouche-Gaudron C, editor. Conjugalité et Parentalité. Ramonville-SaintAgne: Erès; 2005. Coll. Mille et un bébés, p.37-71.

[25] Weissman SH, Cohen RS. The parenting alliance and adolescence. Adolesc Psychiatr 1985;12:24-45.

[26] Waters E, Deane KE. Defining and assessing individual differences in attachment relationships: Q-methodology and the organization of behavior in infancy and early childhood. In: I. Bretherton and E. Waters (editors.), Growing points of attachment theory and research. Monographs of the Soc Res Child Dev, 1985, 50 (1-2), S209: 41-65.
Delachaux et Niestlé; 1991.

[29] Nair H, Murray AD. Predictors of attachment security in preschool children from intact and divorced families. J Genet Psychol 2005;166:24563.

[30] Karavasilis L, Doyle AB, Markiewicz D. Associations between parenting style and attachment to mother in middle childhood and adolescence. Int J Behav Dev 2003;27:153-65.

[31] Lamborn SD, Mounts NS, Steinberg L, Dornbusch SM. Patterns of competence and adjustment among adolescents from authoritative, authoritarian, indulgent and neglectful families. Child Dev 1991;62:104965.

[32] Kerns KA, Keplac L, Cole AK. Peer relationships and preadolescents' perceptions of security in the mother-child relationships. Dev Psychol 1996;32:457-66.

[33] Richaud de Minzi MC. Loneliness and depression in middle and late childhood: the relationship to attachment and parental styles. J Genet Psychol 2006;167:189-210.

[34] Claës M, Lacourse E. Pratiques parentales et comportements déviants à 1'adolescence. Enfance 2001;4:379-99.

[35] Rauer AJ, Volling BL. Differential parenting and sibling jealousy: Developmental correlates of young adults' romantic relationships. Pers Relationships remplacer par:

[36] Ferring D, B Eqleichbehandlung in kindheit und Erwachsenenalters ttlerentErwashsenenalters. Parental differential treatment in childhood and adolescence from the perspective of middle-aged adults. Zeitschrift für Entwicklungspsychologie und pädagogische. Psychologie 2003;35:83-97.

[37] Payne KM. Parent gender and attachment security in dual-career couples. Dissertation Abstracts International: Section B. Sci Eng 2001;62:1593.

[38] Bigras M, Paquette D. L'interdépendance entre les sous-systèmes conjugal et parental: une analyse Personne-Processus-Contexte. The interdependence between marital and parental subsystems: A person-process-context analysis. Psicologia: Teoria e Pesquisa 2000;16:91-102.

[39] Sroufe LA, Cooper RG, Dehart GB. Child Development: Its nature and course. New York: McGraw Hill; 1990.

[40] Izard CE, Haynes OM, Chisholm G, Baak K. Emotional determinants of infant-mother attachment. Child Dev 1991;62:905-18.

[41] Chase-Lansdale PL, Owen MT. Maternal employment in a family context: effects on infant-mother and infant-father attachments. Child Dev 1987;58:1505-12.

[42] Schoppe-Sullivan SJ, Diener ML, Mangelsdorf SC, Brown GL, McHale JL, Frosch CA. Attachment and sensitivity in family context: the role of parent and infant gender. Infant Child Dev 2006;15:367-85.

[43] Harrison LJ, Ungerer JA. Maternal employment and infant-mother attachment security at 12 months postpartum. Dev Psychol 2002;38:75873.

[44] Campbell SB, Brownell CA, Hungerford A. The course of maternal depressive symptoms and maternal sensitivity as predictors of attachment security at 36 months. Dev Psychopathol 2004;16:231-52.

[45] Brand H, Crous BH, Hanekom JD. Perceived parental inconsistency as a factor in the emotional development of behaviour disordered children. Psychol Rep 1990;66:620-2.

[46] Scher A. Facilitators and regulators: Maternal orientation as an antecedent of attachment security. J Reprod Infant Psychol 2001;19:325-33.

[47] Szewczyk-Sokolowski M, Bost KM. Attachment, temperament and preschool children's peer acceptance. Blackwell Publishing Ltd; 2005.

[48] Robinson JA, Lille C. Emotional availability in mother-twin dyads: Effects on the organisation of relationships. Psychiatr, Interpers Biol Process 1994;57:222-31.

[49] McBride BA, Schoppe SJ, Rane TR. Child characteristics, parenting stress, and parental involvement: fathers versus mothers. J Marriage Fam 2002;64:998-1011.

[50] Whelan DJ. Using attachment theory when placing siblings in foster care. Child Adolesc Soc Work J 2003;20(1):21-36. 
[51] Stewart R. Sibling attachment relationships: child-infant interactions in the strange situation. Dev Psychol 1983;19(2):192-9.

[52] Teti DM, Ablard KE. Security of attachment and infant-sibling relationships: A laboratory study. Child Dev 1989;60:1519-28.

[53] Troupel O, Zaouche-Gaudron C. Le lien d'attachement fraternel: l'utilisation des histoires à compléter. Rev Int Educ Fam 2004;8(1):27-38.
[54] Volling BL, Belsky J. The Contribution of Mother-Child and Father-Child Relationships to the Quality of Sibling Interaction: A longitudinal study. Child Dev 1992;63:1209-22.

[55] Ainsworth M. Attachment and exploratory behaviour at one-year-old in a strange situation. In: Foss BM, editor. Determinants of Infants Behaviour. London: Methuen; 1969.

Pour citer cet article : Pinel-Jacquemin S, Zaouche-Gaudron C. Système familial et attachement : revue de la question. Neuropsychiatrie de l'enfance et de l'adolescence (2008), doi:10.1016/j.neurenf.2008.09.006 